\title{
A Channel Estimation Method for MIMO-OFDM Mobile WiMax Systems
}

\author{
Fabien Delestre and Yichuang Sun \\ School of Engineering and Technology \\ University of Hertfordshire, Hatfield, Herts, AL10 9AB, UK
}

\begin{abstract}
In this paper, channel estimation for Space-Time Block Code (STBC) - Orthogonal Frequency Division Multiplexing (OFDM) is investigated for Mobile WiMax systems. A new channel estimation approach is proposed using the dedicated pilot subcarriers defined at constant intervals by the WiMax standard. The estimation method has low computation as only linear operations are needed due to orthogonal pilot coding. The performances of the proposed method have been demonstrated by extensive computer simulations. For the OFDM system with two transmit antennas and one to four receive antennas and using QPSK modulation, the simulated results under different Stanford University Interim (SUI) channels show that the proposed method has only a $4 \mathrm{~dB}$ loss compared to the ideal case where the channel is known at the receiver.
\end{abstract}

\section{INTRODUCTION}

In recent years, Mobile WiMax has received wide interest for next generation wireless communications [1, 2]. Two key technologies, Multiple Input Multiple Output (MIMO) and Orthogonal Frequency Division Multiplexing (OFDM) have been adopted in Mobile WiMax standard, IEEE 802.16e (Mobile WiMax) which is an extension of the IEEE 802.162004 [3], which enable high data rate transmission over multipath and frequency selective fading channels. Due to its simple linear decoding, thus a low complexity receiver, Space-Time Block Codes (STBC) and STBC-OFDM are considered for Mobile WiMax communications. Accurate channel state information (CSI) is required to perform coherent detection of STBC-OFDM signals and estimating mobile channels for high data rate communications has proved to be very challenging.

Two approaches for channel estimation have been proposed in the literature. Blind channel estimation which relies on the exploitation of the statistical information of the received symbols [4], has the bandwidth-saving advantage. However, the blind technique is limited to slow time varying channels and has higher complexity at the receiver. On the other hand, pilot aided channel estimation uses pilot sequences sent as a preamble of each data frame and known at the receiver is simple to implement and can be applied to different types of channels although the use of pilots affects the data rate. In this paper we propose a pilot aided channel estimation method for MIMO-OFDM Mobile WiMax systems.

Channel estimation of MIMO-OFDM systems has been widely investigated by researchers $[5,6]$ and two kinds of approache have been introduced, frequency and time domain channel estimation [7, 8]. In these methods, a complete OFDM frame composed of training symbols is first sent in order to estimate the channel parameters. The channel is therefore assumed to be constant for the next OFDM blocks until a new estimation is performed. In fast fading environment, performances degradation would be noticed due to the outdated channel estimation. Normally, estimation performed in time domain would be more appropriate as impulse responses contain fewer parameters than frequency responses.

In this paper, a two-stage channel estimation technique is described. First all the dedicated pilot tones defined in [3] are used to estimate the channel at different intervals in the OFDM block. Once obtained, the channel parameter and OFDM block are then divided in groups which are used for data decoding. Each group of data is allocated to a pilot tone which is first used to estimate the channel parameter and then used to decode the data group. Simulations were conducted based on the specifications given in the IEEE 802.16e standard for mobile WiMax and under Stanford University Interim (SUI) channel models.

The rest of the paper is organised as follows. In Section II, the structure of the proposed algorithm is presented for the simple $2 \times 1$ case of STBC-OFDM for clarity. Section III proposes a channel estimation algorithm with a full description using equations and diagram. In Section IV, performances of the proposed algorithm are demonstrated by computer simulations under various channel environments. Section V concludes the paper.

\section{STBC-OFDM FOR MOBILE WiMAX SYSTEMS}

The proposed STBC-OFDM system with two transmit antennas and one receive antenna is shown Fig. 1. However, as demonstrated in the following section, discussion can easily be extended to higher number of transmit or receive antenna.

From Fig. 1, it can be seen that at a transmission time n, a random data stream is generated, modulated and after pilot insertion, sent to the STBC encoder. The STBC vectors are then processed by an Inverse Fast Fourier Transform (IFFT) and the resulting signals are simultaneously transmitted from the antennas after the addition of the cyclic prefix and guard interval.

At the receiver side, cyclic prefix and guard interval are first removed and then, the received signal is passed through the Fast Fourier Transform (FFT) block. The output of the FFT can be expressed as: 


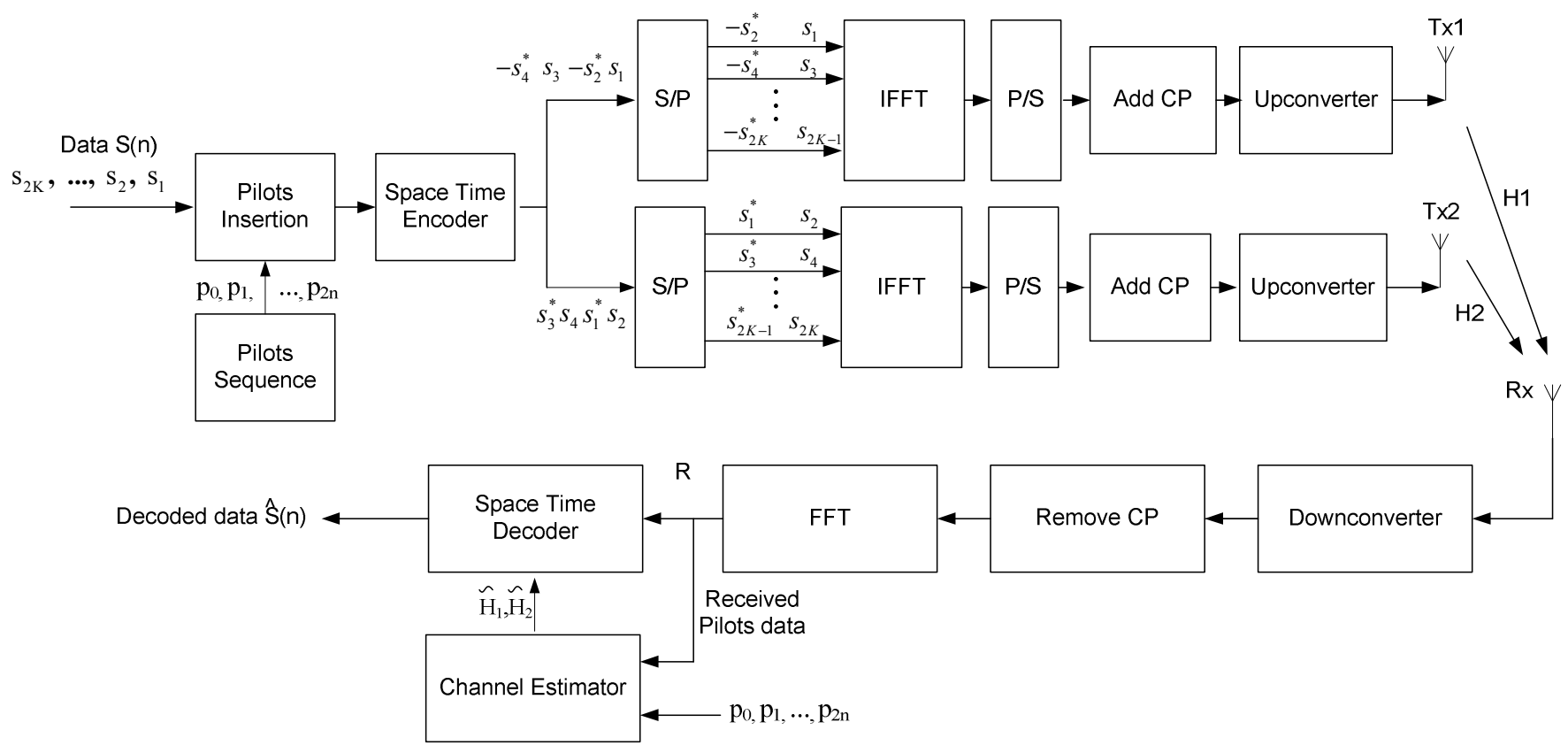

Fig. 1. A block diagram of the proposed MIMO-OFDM Mobile WiMax system

$$
R_{j, k}^{t}=\sum_{i=1}^{n_{t}} H_{i, k}^{t} S_{i, k}^{t}+W_{j, k}^{t}
$$

where $\mathrm{R}_{\mathrm{i}, \mathrm{k}}^{\mathrm{t}}$ is the received signal, $\mathrm{S}_{\mathrm{i}, \mathrm{k}}^{\mathrm{t}}$ is the transmitted signal and $\mathrm{H}_{\mathrm{i}, \mathrm{k}}^{\mathrm{t}}$ is the channel frequency response for the path from the $\mathrm{i}$-th transmit antenna to the $\mathrm{j}$-th receive antenna on the $\mathrm{k}$-th OFDM sub-channel, and $\mathrm{W}_{\mathrm{j}, \mathrm{k}}^{\mathrm{t}}$ represents the noise sample at the $\mathrm{j}$-th receive antenna and at the $\mathrm{k}$-th sub-channel.

Finally, the key for the decoding is channel estimation. Higher number of pilots could be used to obtain more accurate channel estimation. However, increasing the pilot subcarriers would mean less data transmitted and therefore, a nonefficient use of the bandwidth. In the method to be presented below for Mobile WiMax systems, we will use the existing pilot tones in the OFDM format to trade off accuracy of estimation for efficiency of bandwidth.

\section{Proposed ChanNEL Estimation SCHEME}

In this paper, OFDM specifications given by IEEE $802.16 \mathrm{e}$ have been adopted. Each OFDM block is composed of a guard interval, a cyclic prefix, 192 data subcarriers and 8 pilot subcarriers. The OFDM block format from IEEE 802.16 is shown in Fig. 2. As it can be seen, 8 pilot subcarriers are used for 192 data subcarriers. For the bandwidth efficiency, the proposed channel estimation method will use these pilots only.

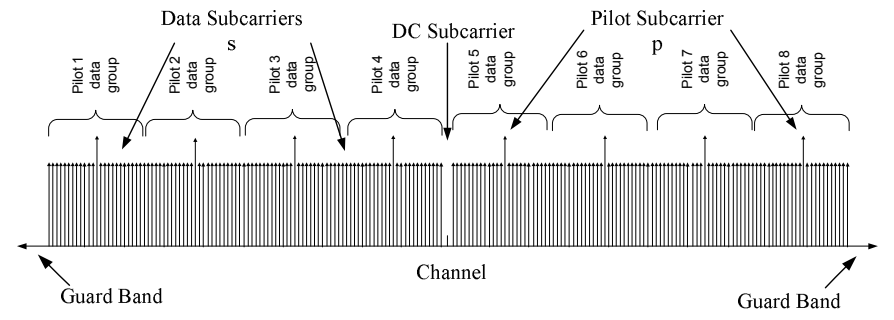

Fig. 2. OFDM block description

We assume that even under Doppler effect, data subcarriers close to each other can be considered as being transmitted over a constant channel. Therefore, the method is grouping part of the data tones together and associates them to one pilot subcarrier. According to the number of pilot and data subcarrier mentioned above, it has been determined that each pilot tone would cover 24 data subcarriers.

As a result, the pilot tone $1,2,3,4,5,6,7$ and 8 are covering the data subcarriers 1:24, 25:48, 49:72, 73:96, 97:120, 121:144, 145:168 and 169:192 respectively. Each group of data is decoded with the channel parameters estimated using the corresponding pilot tone. Hence, if the estimation has not been made accurately due to interference or noise, only part of the OFDM block would not be properly recovered.

As it can be seen from Fig. 3, pilot and data are coded according to space, time and frequency. For clarity reasons, only pilots have been highlighted in Fig. 3 and only part of the OFDM block is described and some data subcarriers between two pilot tones have been omitted.

Pilot sequences are constantly transmitted in order to estimate the channel in a high mobility environment. As the method is based on STBC, channel parameters have been 
assumed constant over two transmitted OFDM blocks. The proposed method needs two time slots to estimate the channel and recover the transmitted data.

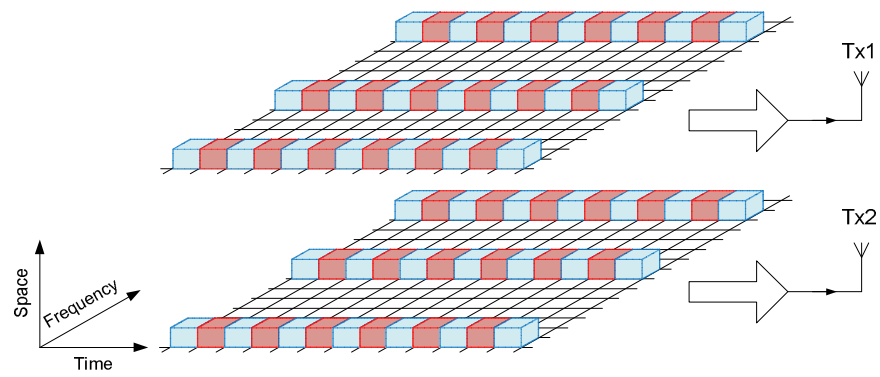

Fig. 3. Organisation of the pilot in space, time and frequency

With the help of Fig. 3, it can be noticed that a full cycle will be done once the two time slots (blue and red) have been completed. This is due to the use of STBC which at the first time slot $\mathrm{t}$, the antennas transmit the nonconjugated data and pilot and then transmit the conjugated part of them at $\mathrm{t}+\mathrm{T}$.

At time $t$, an OFDM block is transmitted simultaneously from each antenna and antennas 1 and 2 transmit pilot sequence $p_{1}$ to $p_{15}$ and $p_{2}$ to $p_{16}$ respectively. Then, at time $t+T$, the conjugated part of the pilot $-p_{2}^{*}$ to $-p_{16}^{*}$ and $p_{1}^{*}$ to $p_{15}^{*}$ are transmitted from antennas 1 and 2 respectively. Thus at the defined pilot position, training symbols are coded according to space time block coding structure, which results in a linear estimation due to the orthogonality.

Data are also transmitted according to STBC and therefore, as it can be seen in Table I for antenna 1 and Table II for antenna 2, nonconjugated part is first sent followed by the conjugated part at $\mathrm{t}+\mathrm{T}$.

TABLE I

ENCODED DATA ACCORDING TO TIME AND SUBCARRIER AT ANTENNA 1

\begin{tabular}{|c|c|c|c|}
\hline Time $^{\text {Subcarrier }}$ & 1 & 2 & $\mathrm{~K}$ \\
\hline $\mathrm{t}$ & $s_{1}$ & $s_{3}$ & $s_{2 K-1}$ \\
\hline $\mathrm{t}+\mathrm{T}$ & $-s_{2}^{*}$ & $-s_{4}^{*}$ & $-s_{2 K}^{*}$ \\
\hline
\end{tabular}

TABLE II

ENCODED DATA ACCORDING TO TIME AND SUBCARRIER AT ANTENNA 2

\begin{tabular}{|c|c|c|c|}
\hline Time Subcarrier & 1 & 2 & $\mathrm{~K}$ \\
\hline $\mathrm{t}$ & $s_{2}$ & $s_{4}$ & $S_{2 K}$ \\
\hline $\mathrm{t}+\mathrm{T}$ & $s_{1}^{*}$ & $s_{3}$ & $s_{2 K-1}^{*}$ \\
\hline
\end{tabular}

Given Table I and II and assuming $\mathrm{H}_{\mathrm{i}, \mathrm{k}}^{\mathrm{t}}=\mathrm{H}_{\mathrm{i}, \mathrm{k}}^{\mathrm{t}+\mathrm{T}}$ the received equation at the FFT output, for the simple case of two transmit and one receive antenna can be expressed as:

$$
R_{k}^{t}=H_{1, k}^{t} S_{1, k}^{t}+H_{2, k}^{t} S_{2, k}^{t}+W_{k}^{t}
$$

where $\mathrm{R}_{\mathrm{k}}^{\mathrm{t}}, \mathrm{S}_{\mathrm{i}, \mathrm{k}}^{\mathrm{t}}$ and $\mathrm{W}_{\mathrm{k}}^{\mathrm{t}}$ are the received symbols, transmitted vector symbols and the Gaussian noise sample respectively; $t$ refers to the $\mathrm{t}$-th symbol period and $\mathrm{k}$ to the $\mathrm{k}$-th subcarrier as stated previously.

Moreover, the transmitted symbols, before the IFFT for antenna 1 and 2 at time $t$ and $\mathrm{t}+\mathrm{T}$ can be expressed as:

$$
\begin{gathered}
S_{1, k}^{t}=\left[s_{1}, s_{3}, \ldots, s_{23}, p_{1}, \ldots, s_{2 K-1}\right] \\
S_{1, k}^{t+T}=\left[-s_{2}^{*},-s_{4}^{*}, \ldots,-s_{24}^{*},-p_{2}^{*}, \ldots,-s_{2 K}^{*}\right] \\
S_{2, k}^{t}=\left[s_{2}, s_{4}, \ldots, s_{24}, p_{2}, \ldots, s_{2 K}\right] \\
S_{2, k}^{t+T}=\left[s_{1}^{*}, s_{3}^{*}, \ldots, s_{23}^{*}, p_{1}^{*}, \ldots, s_{2 K-1}^{*}\right]
\end{gathered}
$$

where $\mathrm{p}_{\mathrm{i}}$ represent the training signal. As stated previously, the number of subcarrier per OFDM frame reserved for data and pilots, defined by the standard, are 192 and 8 respectively.

At the receiver, the signal is first demodulated by an FFT and then data are recovered by the space time decoder. For an ideal transmission where the channel is known at the receiver and according to the equations given in [9] for single carrier system, it can be derived for multicarriers:

$$
\begin{gathered}
\tilde{\boldsymbol{S}}_{i, k}=H_{1, k}^{*} r_{k}^{t}+H_{2, k}\left(r_{k}^{t+T}\right)^{*} \\
\tilde{\boldsymbol{S}}_{i+1, k}=H_{2, k}^{*} r_{k}^{t}-H_{1, k}\left(r_{k}^{t+T}\right)^{*}
\end{gathered}
$$

with $\mathrm{k}=1,2, \ldots, 2 \mathrm{~K}$, representing the symbol number and where $\mathrm{K}=192$, i represent the $\mathrm{i}$-th transmit antenna and $\tilde{S}$ is the decoded signal.

In conventional pilot aided channel estimation, pilots are first transmitted in order to estimate the channel. In the proposed method stated below, data and pilot are mixed in an OFDM frame and once the entire frame is received and FFT demodulated, channel is estimated using the pilots in it. Once the channel is estimated, equations (7) and (8) are used to decode the transmitted symbols by replacing $\mathrm{H}_{1}$ and $\mathrm{H}_{2}$ with the estimated ones. Thus, the two received pilot symbols and the corresponding training symbols of each antenna are used to estimate $\mathrm{H}_{1}$ and $\mathrm{H}_{2}$.

In order to estimate the channel, pilot symbols are extracted from the received frame. Therefore, assuming $\mathrm{H}_{\mathrm{i}, \mathrm{n}}^{\mathrm{t}}=\mathrm{H}_{\mathrm{i}, \mathrm{n}}^{\mathrm{t}+1}$, two vectors results of this extraction, one receive data vector and one receive training symbols vector described as $\mathrm{R}_{\mathrm{k}}^{\mathrm{t}}$ and $\mathrm{Rp}_{\mathrm{n}}^{\mathrm{t}}$ given by equations (2) and (9) respectively. 


$$
R p_{n}^{t}=H_{1, n}^{t} P_{1, n}^{t}+H_{2, n}^{t} P_{2, n}^{t}+W_{n}^{t}
$$

where $\mathrm{P}_{\mathrm{i}, \mathrm{t}}^{\mathrm{t}}$ corresponds to the transmitted pilot vector from $\mathrm{i}$-th antenna and $n=1, \ldots 8$ corresponds to the pilot subcarrier number in the pilot vector. The value of $\mathrm{n}$ is limited to 8 as 8 pilot subcarriers have been defined in the standard. Pilot vectors for antennas, 1 and 2 are given below for time $t$ and $\mathrm{t}+\mathrm{T}$.

$$
\begin{aligned}
& P_{1, n}^{t}=\left[\begin{array}{ll}
p_{1} & p_{3}, \ldots, p_{15}
\end{array}\right] \\
& P_{1, n}^{t+T}=\left[-p_{2}^{*}-p_{4}^{*}, \ldots,-p_{16}^{*}\right] \\
& P_{2, n}^{t}=\left[\begin{array}{ll}
p_{2} & p_{4}, \ldots, p_{16}
\end{array}\right] \\
& P_{2, n}^{t+T}=\left[\begin{array}{ll}
p_{1}^{*} & p_{3}^{*}, \ldots, p_{15}^{*}
\end{array}\right]
\end{aligned}
$$

where $\mathrm{P}_{\mathrm{i}-\mathrm{th}}$ refer to the pilot vector of the $\mathrm{i}$-th antenna.

The proposed equations for channel estimation can be found in equations (14) and (15).

$$
\begin{aligned}
& \hat{H}_{1, n}^{t}=\frac{R p_{n}^{t} P_{1, n}^{*}-R p_{n}^{t+T} P_{2, n}}{\left|P_{1, n}\right|^{2}+\left|P_{2, n}\right|^{2}} \\
& \hat{H}_{2, n}^{t}=\frac{R p_{n}^{t} P_{2, n}^{*}+R p_{n}^{t+T} P_{1, n}}{\left|P_{1, n}\right|^{2}+\left|P_{2, n}\right|^{2}}
\end{aligned}
$$

Once the channels have been estimated using equations (14) and (15), channel parameters in the decoding formulas in equations (7) and (8) are replaced by the estimated ones in order to recover all the data in the transmitted OFDM block. Due to the fact that 8 pilot tones have been used, 8 channel estimations will result from equations (14) and (15). These estimations are used to decode the corresponding groups of data as described earlier.

By replacing $\mathrm{H}_{1}$ and $\mathrm{H}_{2}$ in equations (7) and (8) by $\hat{H}_{1}$ and $\hat{H}_{2}$ in equations (14) and (15), it can be deduced:

$$
\begin{gathered}
\tilde{\boldsymbol{S}}_{i, k}=\hat{H}_{1, k}^{*} r_{k}^{t}+\hat{H}_{2, k}\left(r_{k}^{t+T}\right)^{*} \\
\tilde{\boldsymbol{S}}_{i+1, k}=\hat{H}_{2, k}^{*} r_{k}^{t}-\hat{H}_{1, k}\left(r_{k}^{t+T}\right)^{*}
\end{gathered}
$$

where $\hat{H}_{1}$ and $\hat{H}_{2}$ contain the channel parameters of different groups.

As it can be noticed from the above derivations, the proposed channel estimation method is very simple and therefore is cost and computation effective, because the channel is estimated using only eight pilot tones which allow the receiver to decode the entire OFDM frame and no matrix inversion is required at the receiver for the channel to be estimated. Allocating a group of subcarrier to a pilot tone slightly increases the complexity but the method is still cost effective due to its simplicity at the receiver. Moreover, at the transmitter side, only pilot sequence needs to be added to the transmitted signal.

\section{IV.PeRformance EVAluation}

The performances of the proposed channel estimation algorithm are evaluated by computer simulations in a Mobile WiMax environment. Mobile channels used for the evaluation of the performance, are multipath channels each experiencing Rayleigh fading with Doppler frequency. The parameters of the channel were set according to the definition of the SUI standard.

For the simulations of the algorithm, a two transmit antennas with one, two, three and four receive antennas have been used. In addition, as stated earlier, an OFDM block of 256 subcarriers defined in the Mobile WiMax standard have been used which includes 192 data tones, 8 pilot tones and 56 guard tones. Performances were observed under SUI channels 1 and 3 for QPSK modulation. The channel bandwidth was set to $2 \mathrm{MHz}$ and the OFDM block time to $115 \mu \mathrm{s}$ with a guard interval of $14 \mu \mathrm{s}$. The performances of the proposed algorithm are measured in term of Bit Error Rate (BER) versus Signal to Noise Ratio (SNR).

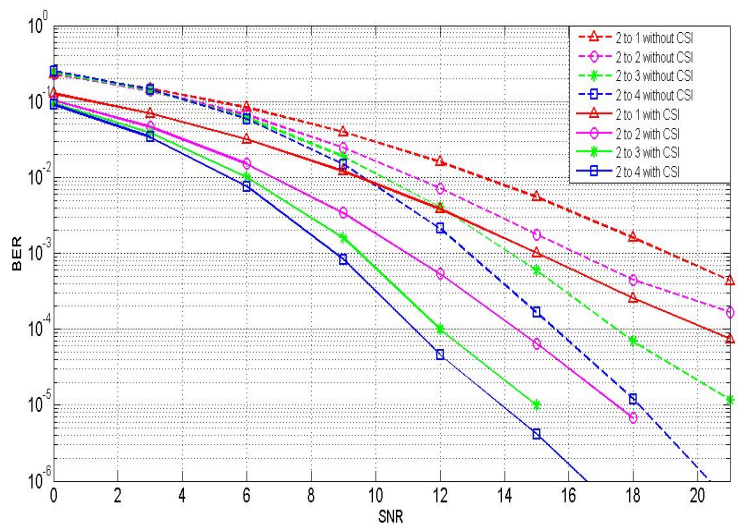

Fig. 4. Performance of the system under channel model SUI1

Fig. 4 shows the performance of the algorithm for two transmit antennas and one to four receive antennas evaluated under channel model SUI1 and using QPSK modulation. It can be seen that higher number of receive antennas decrease the value of the BER. In addition, the proposed algorithm in the four different cases has a $4 \mathrm{~dB}$ loss between the ideal case where the channel is known at the receiver and the proposed channel estimation technique. This is a satisfying result given the small number of pilots used. 


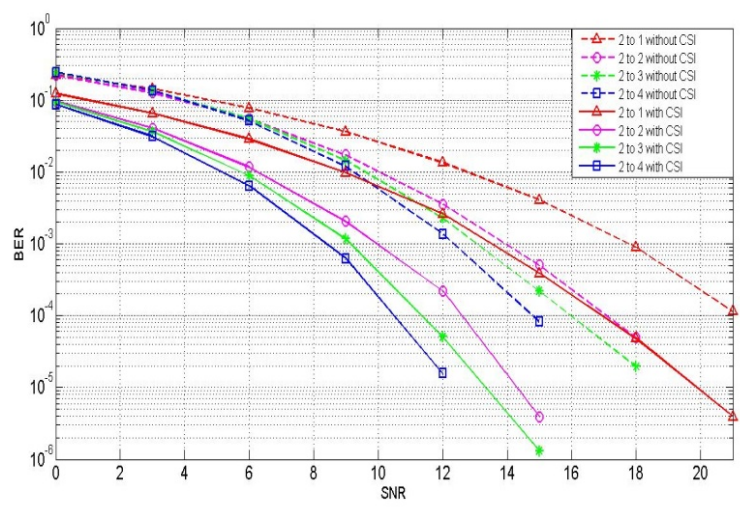

Fig. 5. Performance of the system under channel model SUI3

Fig. 5 plots the performance for two transmit antennas and one to four receives antennas under SUI3 and using QPSK. As stated for the SUI1 case, the performance improves when the number of receive antenna increases. The channel estimation method under SUI3 is also giving a $4 \mathrm{~dB}$ penalty, which proves that the proposed method is suitable for mobile channels.

Simulations using higher order of modulation and higher number of transmit antenna can also be conducted.

\section{CONCLUSION}

In this paper, an efficient channel estimation technique for MIMO-OFDM communications and particularly for Mobile WiMax systems has been proposed. The method requires low computation as matrix inversion is not required at the receiver due to the orthogonal pilot coding used and only uses the existing pilot tones in the standard IEEE 802.16e. The overall performance of the proposed algorithm has been evaluated by computer simulations. For MIMO-OFDM systems using two transmit antennas under various SUI channel conditions, the proposed method has a penalty of only $4 \mathrm{~dB}$ for the small number of pilots compared with the ideal case where the channel is known at the receiver.

\section{REFERENCES}

[1] T. Koon Hoo, T. Zhifeng, Z. Jinyun, "The Mobile Broadband WiMAX Standard [Standards in a Nutshell]", IEEE Signal Processing Magazine, vol. 24, pp. 144-148, 2007.

[2] K. Etemad, "Overview of mobile WiMAX technology and evolution", IEEE Communications Magazine, vol. 46, pp. 31-40, 2008.

[3] "IEEE recommended practice for local and metropolitan area networks. Coexistence of fixed broadband wireless access systems", IEEE Std 802.16.2-2004 (Revision of IEEE Std 802.16.2-2001), 2004.

[4] M H. Bolcskei, R. W. Heath, Jr. and A. J. Paulraj, "Blind equalization in OFDM-based multi-antenna systems", in The IEEE 2000 Adaptive Systems for Signal Processing, Communications, and Control Symposium, pp. 58-63, 2000.

[5] L. Ye, N.Seshadri and S. Ariyavisitakul, "Channel estimation for OFDM systems with transmitter diversity in mobile wireless channels", IEEE Journal on Selected Areas in Communications, vol. 17, pp. 461471, 1999.

[6] W. Feng, W. P. Zhu and M. N. S. Swamy, "A Semiblind Channel Estimation Approach for MIMO-OFDM Systems", IEEE Transactions on Signal Processing, vol. 56, pp. 2821-2834, 2008.

[7] H. Y. Chen, M. L. Ku, S. J. Jou and C. C. Huang, "A Robust Channel Estimator for High-Mobility STBC-OFDM Systems", IEEE Transactions on Circuits and Systems I: Regular Papers, vol. 57, pp. 925-936, 2010.

[8] M. Morelli and U. Mengali, "A comparison of pilot-aided channel estimation methods for OFDM systems", IEEE Transactions on Signal Processing, vol. 49, pp. 3065-3073, 2001.

[9] S. M. Alamouti, "A simple transmit diversity technique for wireless communications", IEEE Journal on Selected Areas in Communications, vol. 16, pp. 1451-1458, 1998. 